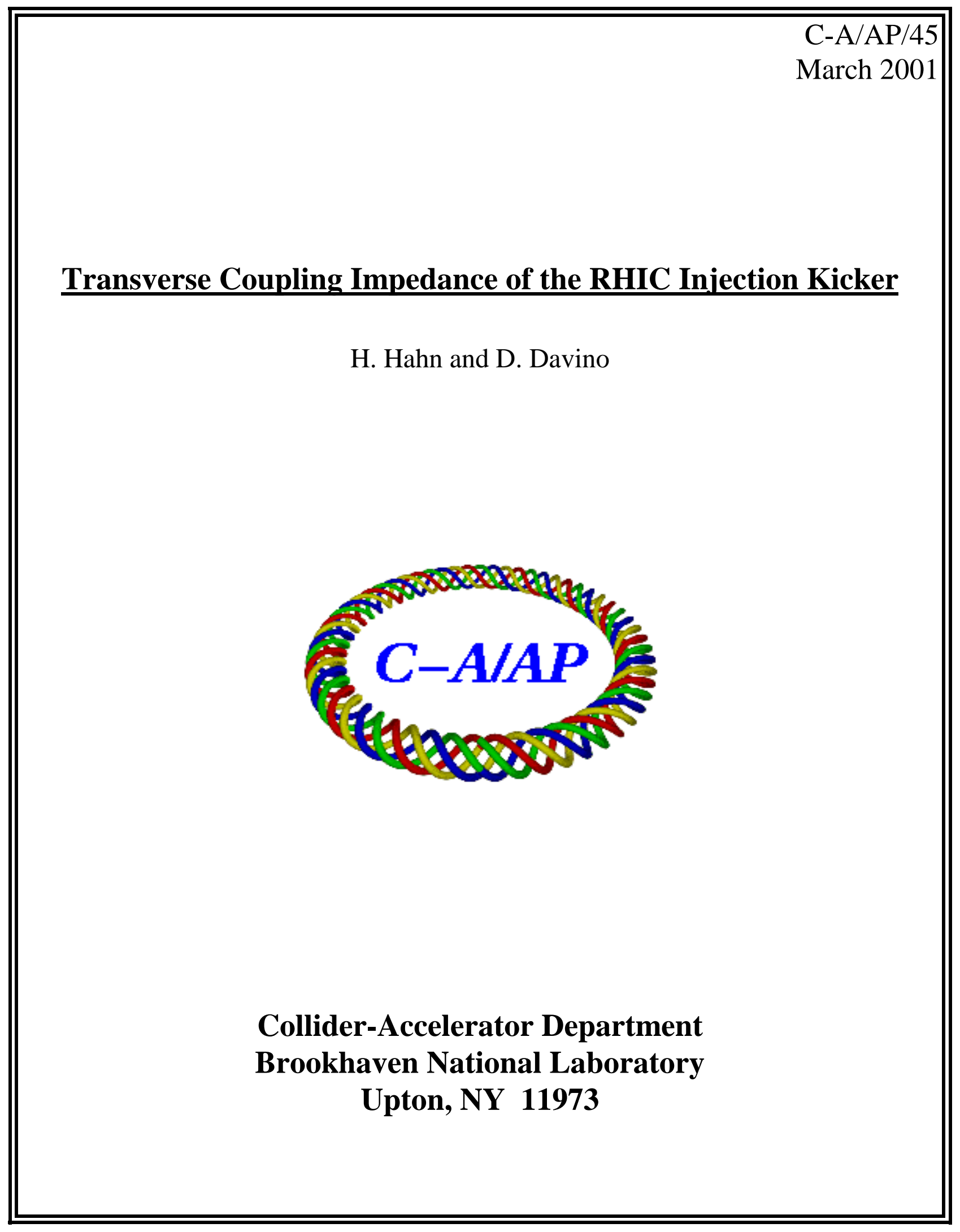




\title{
Transverse Coupling Impedance of the RHIC Injection Kicker
}

\author{
H. Hahn and D. Davino
}

\begin{abstract}
The results of transverse coupling impedance measurements of the RHIC injection kickers are presented. The impedance measurements were made using the standard twowire method to obtain the forward transmission coefficient $S_{21}$ of the "Device Under Test" with respect to a reference tube of equal length, and were interpreted via the logformula. The measurements were made in a test stand, reflecting fully the operational conditions. The transverse impedance of the dielectric loaded traveling wave kicker is largest below $10 \mathrm{MHz}$ and reaches for a single unit in the vertical, i.e. the kick direction $190+\mathrm{j} 350 \mathrm{k} \Omega / \mathrm{m}$ and in horizontal direction $100+\mathrm{j} 300 \mathrm{k} \Omega / \mathrm{m}$. The inductive component of the all-ferrite version is about $20 \%$ larger. The contribution to the total impedance budget reflects four kicker units per ring.
\end{abstract}

\section{Introduction}

Performance Limitations in RHIC due to collective effects were originally studied in the 1988 Workshop $^{1}$ and are discussed in the May 1989 Conceptual Design Report ${ }^{2}$. Since instabilities depend on ion mass number, $A$, and charge, $Q$, as $A / Q^{2}$, it was assumed that the impedance contributions can be held to a level that would not limit the heavy ion performance but may well limit the use of RHIC as a high-luminosity proton storage ring. The subsequent review of collective instabilities ${ }^{3,4}$ confirmed these conclusions and a program to control impedance contributions was implemented. Limits on the longitudinal impedance are primarily given by the passage though transition and limits on transverse impedance by the so-called resistive wall instability at injection.

Major contributors to the transverse coupling impedance are the resistive wall, and the injection and abort kickers. The textbook expression for the transverse resistive wall impedance, based on Panofsky-Wenzel scaling of the longitudinal impedance, is ${ }^{5}$

$$
Z_{\perp}=2 \frac{R}{b^{2}} \frac{Z_{\|}}{n}=(1+j) \frac{Z_{0} R}{b^{3}} \delta_{s}
$$

with $b$, the radius of the circular beam tube, $2 \pi R$ the collider circumference, and the skin depth

$$
\delta_{s}=\sqrt{2 \rho / \mu_{0} \omega} .
$$

The beam tube in RHIC is stainless with radii varying from $\sim 7 \mathrm{~cm}$ in the $3 \mathrm{~km}$ long arc magnets to $\sim 12 \mathrm{~cm}$ in the $800 \mathrm{~m}$ long warm sections. The resistive wall contribution to the transverse coupling impedance in the "two pipe model ${ }^{3 "}$ is estimated to be

$$
Z_{\perp} \approx \frac{5.88}{\sqrt{n}} \mathrm{M} \Omega / \mathrm{m}
$$

with the mode number $n=\omega R / c$. The impedance $Z_{\perp}(n=1)$ at the revolution frequency, $\sim 78 \mathrm{kHz}$, serves as a reference point for comparison with contributions from other ring components, e.g. in our case the injection kickers. 
The relation between beam and the kicker, (assumed electrically short, i.e. at low frequencies) can be approximated as a transformer, for which one finds

$$
Z_{\|}=j \omega L_{B}+\frac{\omega^{2} M^{2}}{j \omega L_{K}+Z_{g}}
$$

with the mutual inductance $M^{2}=\kappa^{2} L_{B} L_{K}$, the kicker inductance $L_{K}$, and the external load impedance $Z_{g}$, and $L_{B}$ the inductance seen by the beam in the absence of an external load. The coupling coefficient depends on the kicker aspect ratio height over width, $h / w$, and could in principle be measured; a first order estimate is $\kappa^{2}=0.5$. Applying the Panofsky-Wenzel scaling together with dimensional analysis leads to an order of magnitude estimate for the transverse coupling impedance of

$$
Z_{\perp} \sim j \frac{Z_{0} l}{w h}\left(1-\frac{1}{2\left[1-j Z_{g} / \omega L_{K}\right]}\right)
$$

with $w$ the width and $h$ the height in kick-direction, and $l$ the kicker length. For the unterminated, $Z_{g}=\infty$, RHIC injection kicker ${ }^{6}$ with $w=48.4 \mathrm{~mm}, h=47.6 \mathrm{~mm}$, and $l=$ $1.12 \mathrm{~m}$ one finds by ignoring the second term in the parenthesis the transverse impedance in vertical and also in horizontal direction to be $Z_{\perp} \sim 180 \mathrm{k} \Omega / \mathrm{m}$.

Estimates, also obtained by Panofsky-Wenzel scaling, for the transverse impedance of traveling wave kickers are given in the Handbook as ${ }^{7}$

$$
Z_{\perp}=\frac{Z_{c} l}{h w}\left[\frac{1-\cos \Theta}{\Theta}+j\left(1-\frac{\sin \Theta}{\Theta}\right)\right]
$$

with the $Z_{c}$ the characteristic impedance and the electrical length

$$
\Theta=\frac{h Z_{0}}{w Z_{c}} \frac{\omega l}{c}
$$

For the RHIC injection kicker, with $Z_{c}=25 \Omega$, follows in the low frequency limit $Z_{\perp} \rightarrow 0$ and an upper limit of $Z_{\perp} \approx 12.2 \mathrm{k} \Omega / \mathrm{m}$. This estimate is of limited value since it assumes fully matched loads and gives only results for the transverse impedance in the kick-direction and ignores the perpendicular direction. In any case, this expression is qualitatively different from the experimental results shown below.

Detailed measurements of the longitudinal coupling impedance were made on the RHIC injection kicker which gave the results for 4 units per ring as ${ }^{8} Z_{\|} / n=0.22 \Omega$ which by scaling ${ }^{9}$ (using $\mathrm{h} / 2$ instead of $b$ ) nominally corresponds to the transverse impedance value of $Z_{\perp} \simeq 120 \mathrm{k} \Omega / \mathrm{m}$ per unit at frequencies up to $100 \mathrm{MHz}$. Although this suggests that the injection kicker is negligible compared to the resistive beam tube, in view of the error margin in the estimates, direct measurements of the transverse impedance are fully justified. 


\section{Transverse Impedance Measurements}

Transverse impedance measurements on the RHIC injection kicker were made using the standard method, ${ }^{10}$ in which a double-wire "Lecher" line is inserted into the kicker and the forward transmission coefficients $S_{21}$ of the "Device Under Test" and of a reference line of equal length is interpreted according to

$$
Z_{\text {DUT }} \approx-2 Z_{L} \ln \left(S_{21 D U T} / S_{21 R E F}\right)
$$

from which one obtains the transverse impedance as ${ }^{11}$

$$
Z_{\perp}=\frac{c Z_{D U T}}{\omega \Delta^{2}}=-2 \frac{c Z_{L}}{\omega \Delta^{2}} \ln \left(S_{21 D U T} / S_{21 R E F}\right)
$$

with $\Delta$ being the spacing of the two wires and $Z_{L}$ the characteristic impedance of the line. In order to obtain sufficiently strong signals, first measurements with the conventional $300 \Omega$ TV cable were replaced with a commercial $450 \Omega$ "Ladder" cable CQ551, which has a spacing of $\Delta=13 / 16 \mathrm{in} .=2.064 \mathrm{~cm}$. Matching of the $450 \Omega$ line to the $50 \Omega$ of the network analyzer, Agilent $8753 \mathrm{ES}$, is achieved by means of a transformer with a centertapped secondary winding which serves as $180^{\circ}$ hybrid. Common mode suppression at frequencies up to $\sim 100 \mathrm{MHz}$ was verified by measuring $Z_{D U T}$ with the line rotated by $180^{\circ}$ and all results quoted represent the average of these two measurements. All measurements were made with the network analyzer set for a logarithmic frequency scale from $30 \mathrm{kHz}$ to $100 \mathrm{MHz}, 1601$ points, and a $30 \mathrm{~Hz}$ bandwidth. The transformer at the input has a 50/450 ratio whereas the output transformer has a ratio of 300/50 and resistive matching by two $50 \Omega$ resistors was applied. The transformers are rated only for frequencies above $100 \mathrm{kHz}$, and measurement results at lower frequencies become suspect and must be used with care.

\section{Experimental Results: TW Injection Kicker}

The RHIC dielectric loaded traveling wave injection kicker is fully described in Ref. 6. The measurements here presented were performed with a single unit on the test stand, ready for installation into the ring, i.e. with full-length cable connections to the blumlein pulser at the input, with a $16 \Omega$ termination at the output end, and with a ceramic beam tube. The impedance budget for the ring must take into account that injection requires 4 units per ring. The results for the transverse impedance applicable during RHIC operation of a single unit are shown in Fig. 1 for the vertical kick direction and in Fig. 2 for the horizontal direction. Shown in all figures are only results above $600 \mathrm{kHz}$. Ignoring results below $1 \mathrm{MHz}$, one sees that the transverse impedances are largest in the range below $\sim 10 \mathrm{MHz}$ and upper limits can be estimated as

$$
\begin{aligned}
& Z_{\perp V} \leq(190+j 350) \mathrm{k} \Omega / \mathrm{m} ;<10 \mathrm{MHz}, \\
& Z_{\perp H} \leq(100+j 300) \mathrm{k} \Omega / \mathrm{m} ;<10 \mathrm{MHz} .
\end{aligned}
$$


Note that the transverse impedance remains finite in the limit of low frequencies in contrast to the Handbook predictions. In fact, all results from the present series of measurements yield a qualitatively different behavior at low frequencies, showing significantly higher values than those quoted in previous publications. ${ }^{12,13}$ The lowfrequency dependence of the impedance seems to be similar to the resistive wall impedance.

The effect of the cable termination on the vertical impedance is clearly visible at frequencies up to $\sim 15 \mathrm{MHz}$. Measurements without cable are shown in Figs. 3 and 4. The results without cable represent in effect an upper limit on the impedance if all other parameters are kept unchanged. As expected, the presence of cables it is not observed in the horizontal direction. But it must be emphasized that the horizontal transverse impedance is evidently not negligible and of the same magnitude as the vertical impedance. Well above $15 \mathrm{MHz}$, the transverse impedances become essentially constant and can be summarized as

$$
\begin{aligned}
& Z_{\perp V} \approx(50+j 110) \mathrm{k} \Omega / \mathrm{m} ; \sim 15 \mathrm{MHz}-100 \mathrm{MHz}, \\
& Z_{\perp H} \approx(20+j 200) \mathrm{k} \Omega / \mathrm{m} ; \sim 10 \mathrm{MHz}-100 \mathrm{MHz} .
\end{aligned}
$$

The contribution from the ceramic beam tube with o.d. of $47.6 \mathrm{~mm}$ and i.d. of $41.3 \mathrm{~mm}$ is best seen in Fig. 3 and Fig. 4. The ceramic is practically lossless and the real part of the transverse impedances is hardly changed, whereas the imaginary part is increased by up to $\sim 50 \mathrm{k} \Omega / \mathrm{m}$ due to the ceramic beam tube.
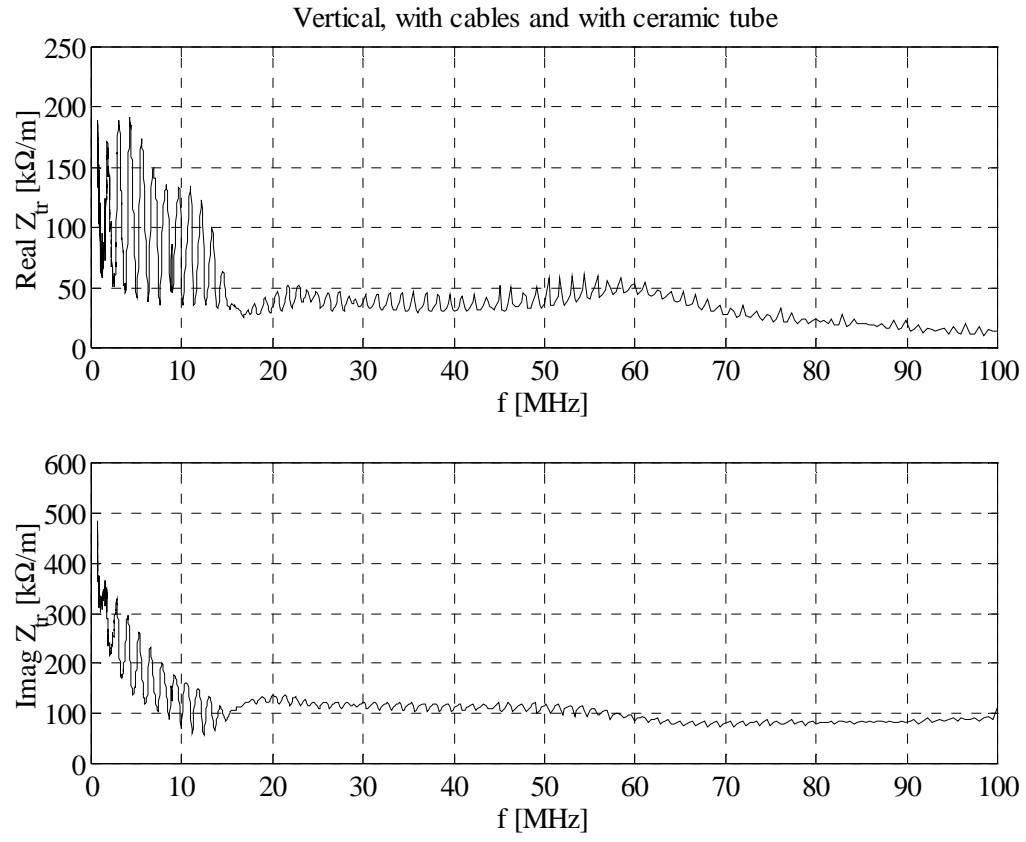

Fig. 1. Vertical transverse impedance of RHIC TW injection kicker 

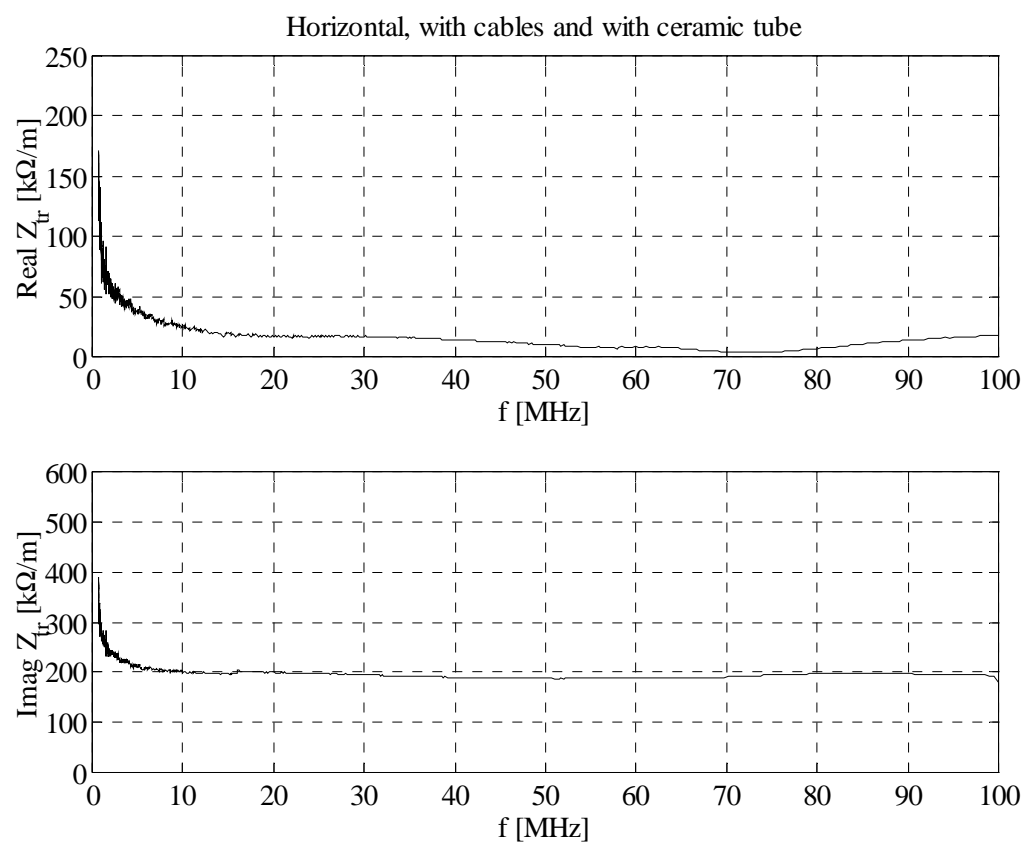

Fig. 2. Horizontal transverse impedance of RHIC TW injection kicker
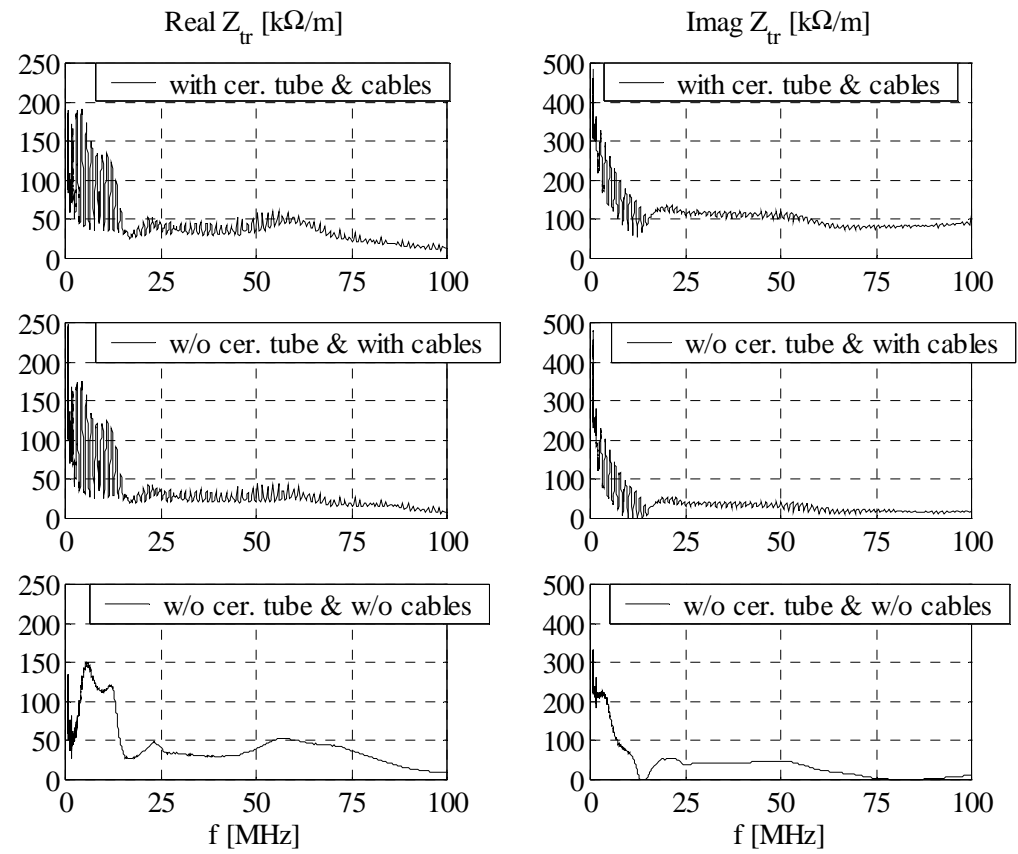

Fig.3. Vertical transverse impedance of TW kicker in different configurations 

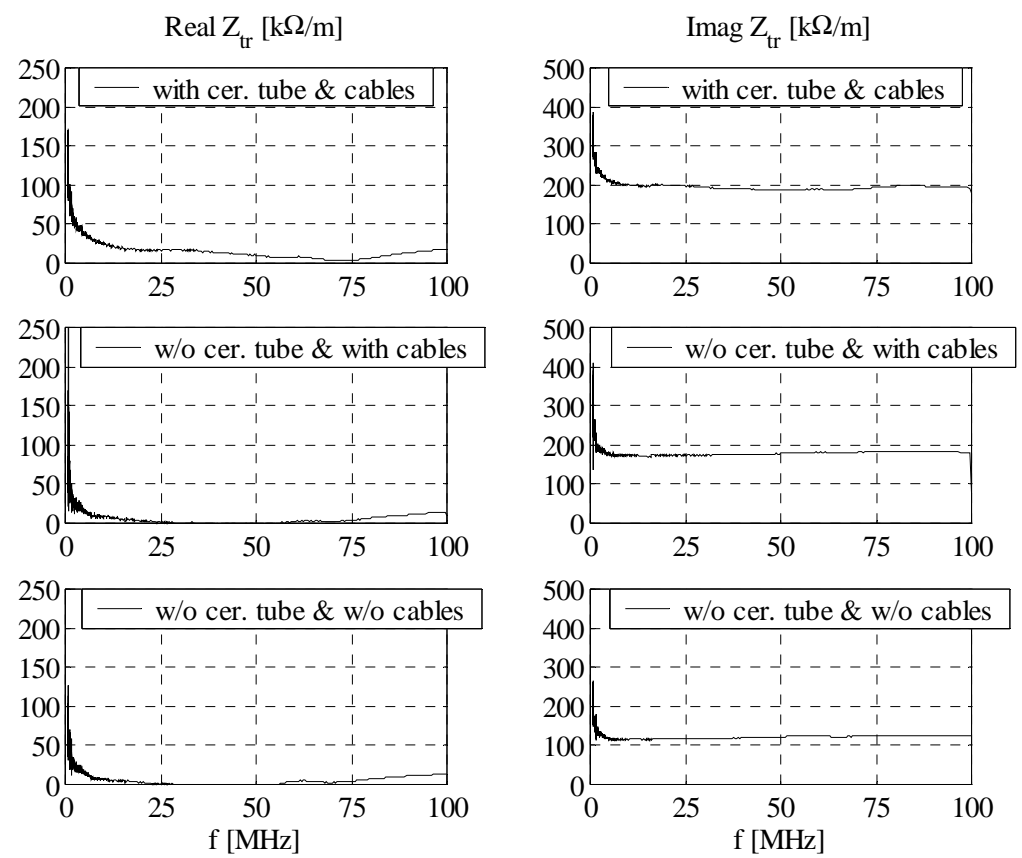

Fig.4. Horizontal transverse impedance of TW kicker in different configurations

\section{Experimental Results: All-Ferrite Injection Kicker}

Future experimental requirements require 120 bunch injection into RHIC and consequently faster kickers. All-ferrite magnets have been installed into the yellow ring and are awaiting confirmation of the expected reduced rise time. The all-ferrite magnet is constructed by replacing the dielectric blocks with ferrite blocks while retaining the overall geometry. As a consequence, the all-ferrite magnet must still be considered as a traveling wave structure, but with a characteristic impedance of $\sim 50 \Omega$ (instead of the $25 \Omega$ ).

Measurements on the all-ferrite kicker were performed in parallel with those on the TW kicker involving the same instruments and kicker test stand. The results are shown in Fig. 5 for the vertical impedance and in Fig. 6 for the horizontal impedance. The more detailed graphs in Fig. 7 and Fig. 8 confirm the similarity of both kicker types. Only the imaginary (inductive) component of the transverse impedances has become about $20 \%$ larger for the all-ferrite magnet. 

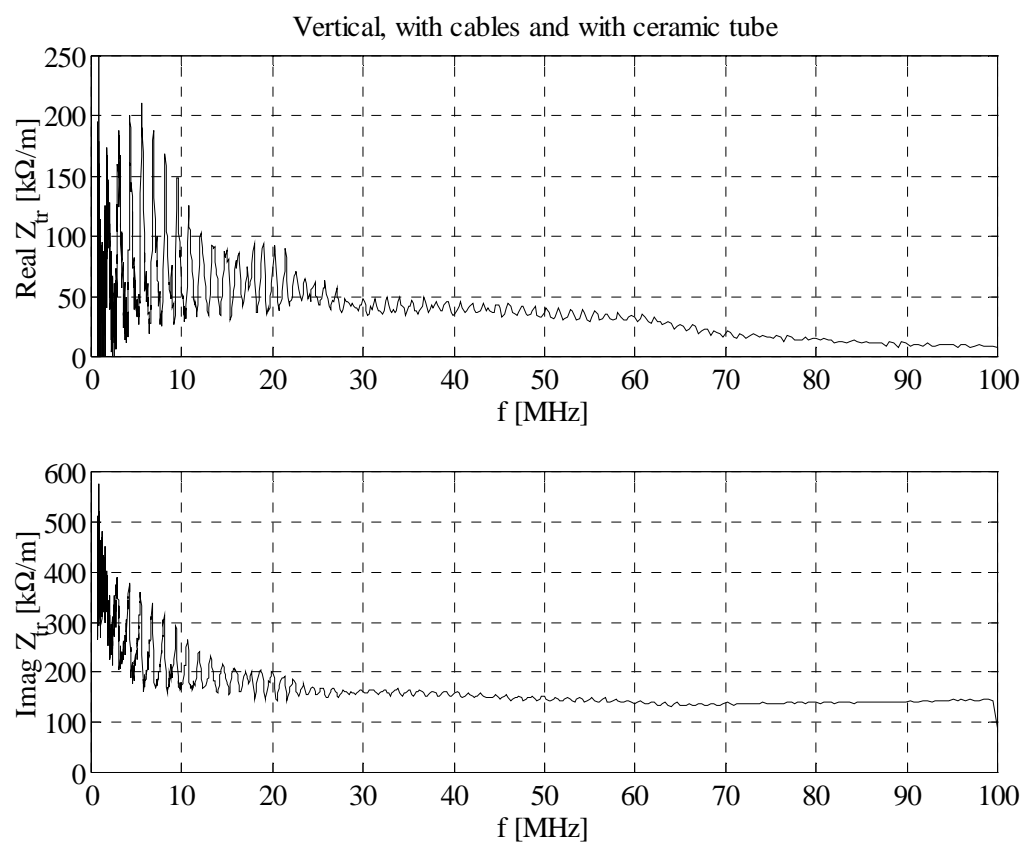

Fig. 5. Vertical transverse impedance of the all-ferrite magnet
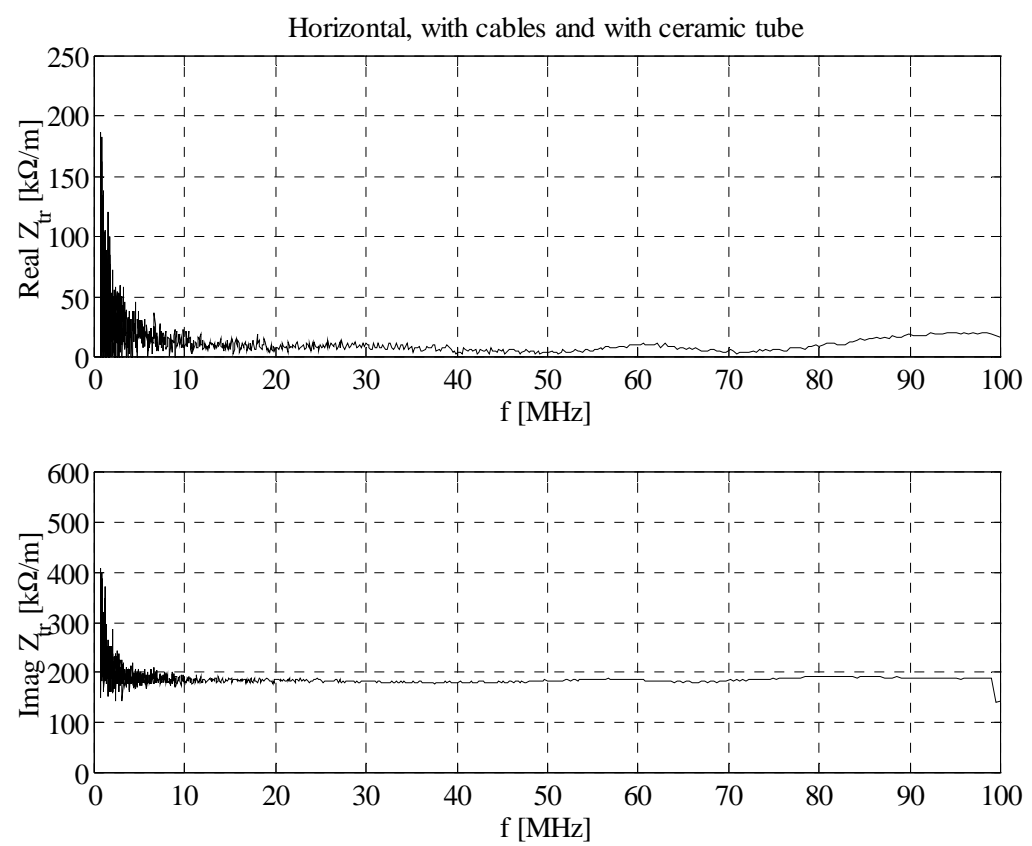

Fig. 6. Horizontal transverse impedance of the all-ferrite magnet. 

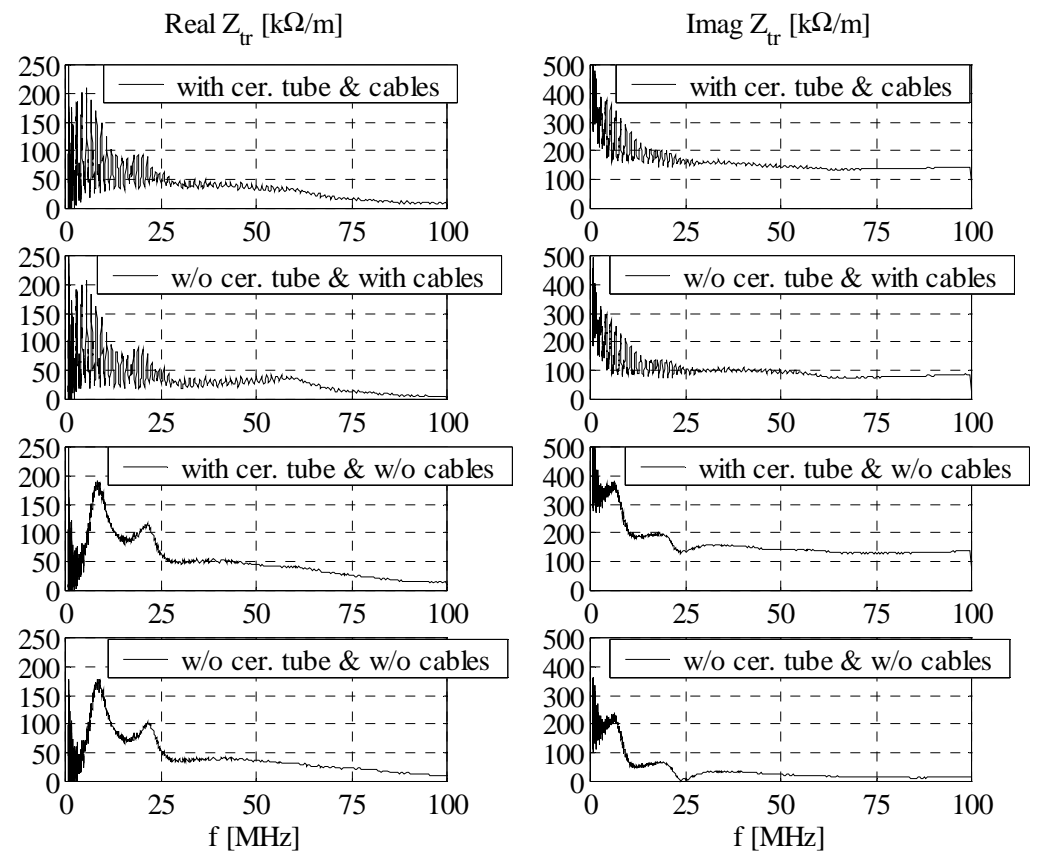

Fig.7. Vertical impedance of all-ferrite kicker in different configurations.
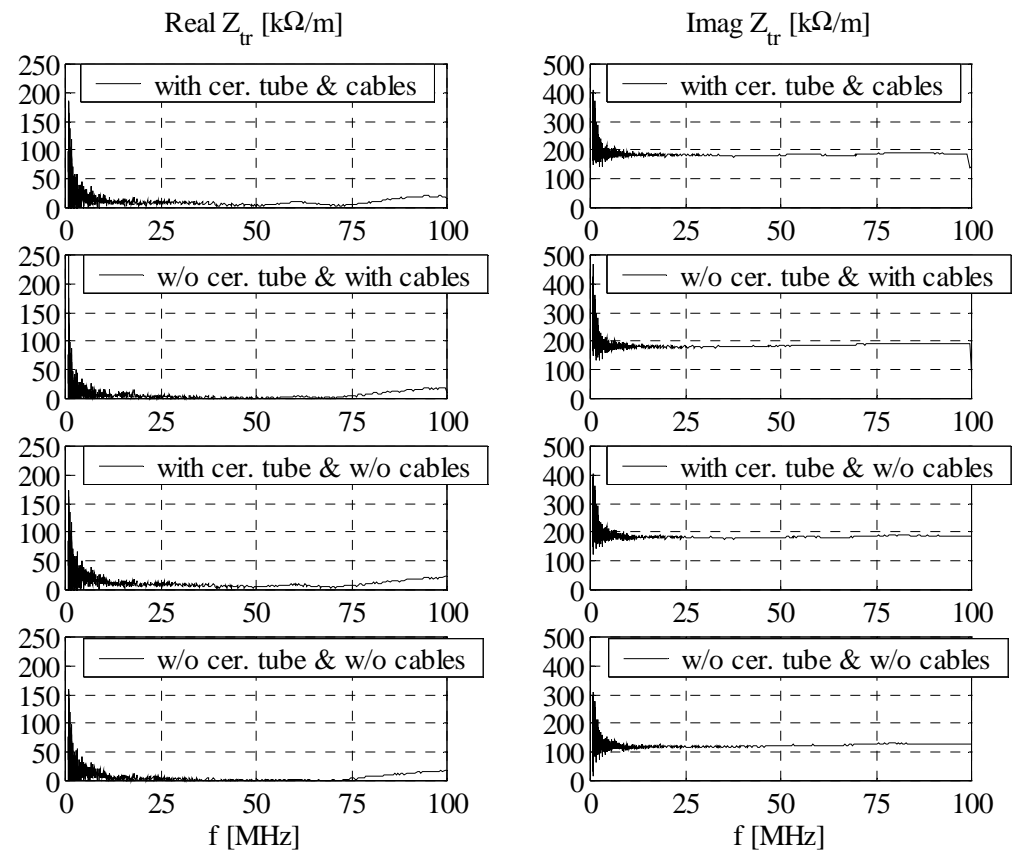

Fig.8 Horizontal impedance of all-ferrite kicker in different configurations. 


\section{Acknowledgements}

The authors would like to thank C. Pai for engineering help, and John Cupolo and Ken Hartmann for valuable technical support.

\section{References}

1. M. S. Zisman, in Workshop on the RHIC Performance, Report BNL-41604, p. 371, (March 1988)

2. Conceptual Design of the Relativistic Heavy Ion Collider, Report BNL 52195, p. 109, (May 1989).

3. S. Peggs and W.W. MacKay, Report RHIC/AP/36, (September 1994)

4. W.W. MacKay, M. Blaskiewicz, D. Deng, V. Mane, S. Peggs, A. Ratti, J. Rose, T.J. Shea, J. Wei, Proc. 1995 PAC, Dallas, TX, p. 3131.

5. B. Zotter, in Handbook of Accelerator Physics and Engineering, ed. A.W. Chao and M. Tigner, p.118.

6. H. Hahn, N. Tsoupas, J. E. Tuozzolo, Proc. 1997 PAC, Vancouver, BC, p. 213

7. K.Y. Ng, in ref. 5, p. 206.

8. H. Hahn and A. Ratti, in ref. 6, p. 1706.

9. B.W. Zotter and S.A. Kheifets, Impedances and Wakes in High-Energy Particle Accelerators, (World Scientific, Singapore, 1998), p. 325.

10. F. Caspers, in ref. 5, p. 571.

11. G. Nassibian and F. Sacherer, Nucl. Instr. Methods, 159, 21 (1979)

12. U. Blell, Proc. 1997 PAC, Vancouver, BC, p. 1727.

13. J.G. Wang and S.Y. Zhang, Proc. 2000 EPAC, Vienna, Austria, p. 972. 